\title{
The gender difference on the Mental Rotations test is not due to performance factors
}

\author{
MARY S. MASTERS \\ San Jose State University, San Jose, California
}

\begin{abstract}
Men score higher than women on the Mental Rotations test (MRT), and the magnitude of this gender difference is the largest of that on any spatial test. Goldstein, Haldane, and Mitchell (1990) reported finding that the gender difference on the MRT disappears when "performance factors" are controlledspecifically, when subjects are allowed sufficient time to attempt all items on the test or when a scoring procedure that controls for the number of items attempted is used. The present experiment also explored whether eliminating these performance factors results in a disappearance of the gender difference on the test. Male and female college students were allowed a short time period or unlimited time on the MRT. The tests were scored according to three different procedures. The results showed no evidence that the gender difference on the MRT was affected by the scoring method or the time limit. Regardless of the scoring procedure, men scored higher than women, and the magnitude of the gender difference persisted undiminished when subjects completed all items on the test. Thus there was no evidence that performance factors produced the gender difference on the MRT. These results are consistent with the results of other investigators who have attempted to replicate Goldstein et al.'s findings.
\end{abstract}

The gender difference on the Mental Rotations test (MRT) is well established (Halpern, 1992; Linn \& Petersen, 1985; Maccoby \& Jacklin, 1974). Men score significantly higher than women on the MRT, and the magnitude of the gender difference is the largest of that on any spatial test (Halpern, 1992; Linn \& Petersen, 1985; Sanders, Soares, \& D'Aquila, 1982). Moreover, the magnitude of the gender difference has remained constant over time (Masters \& Sanders, 1993).

Yet although the evidence for a male advantage is well documented, some investigators have argued that this gender difference is due not to a gender difference in spatial ability, but to "performance factors" on the test that favor men more than women (Goldstein, Haldane, \& Mitchell, 1990). In an article in this journal Goldstein et al. (1990) maintained that the gender difference on the MRT arises not because women make more errors on the test than men do, but because women, working more slowly and cautiously on the test, attempt fewer test items. These investigators contended that in past studies, women performed more poorly on the MRT because the time limit on the test was short and because the scoring procedure took into account only the number of correct responses.

Examination of Goldstein et al.'s (1990) two studies, however, reveals little support for their hypothesis that the MRT gender difference is due to performance factors

I thank Cherie Collister and Donna Head for helping with the data collection and Geoffrey Loftus for his valuable comments and suggestions. Correspondence concerning this article should be addressed to M. S. Masters, Department of Psychology, San Jose State University, San Jose, CA $95192-0120$ (e-mail: mmasters@sprintmail.com).

-Accepted by previous editor, Geoffrey R. Loftus (see Table 1). In their first study, Goldstein et al. allowed 33 male and 35 female college students $3 \mathrm{~min}$ for each part of the two-part MRT. When they first scored the tests with raw scores, or the total number of correct stimuli (two stimuli are correct for each item on the MRT), they found that men scored significantly higher than women. The effect size of the gender difference, as measured by Cohen's $d$, was .85 , a large effect size (Cohen, 1988).

In order to control for the gender difference in the number of stimuli attempted, Goldstein et al. (1990) then used "ratio scores" (the proportion of correct stimuli in the total number of stimuli attempted) to score the tests. This time, both women and men received high scores, the effect size of the gender difference decreased, and the significant gender difference disappeared (see Table 1). Goldstein et al. interpreted this disappearance of the gender difference as evidence that eliminating the gender difference in the number of stimuli attempted eliminates the gender difference in performance on the MRT. An alternative explanation recognized by Goldstein et al. and addressed in their second study is that the absence of the gender difference with ratio scores was due to a ceiling effect.

In their second study, Goldstein et al. (1990) tested 23 college students of each sex. They again allowed the subjects $3 \mathrm{~min} /$ part, but after the time intervals elapsed, they gave them additional time to go back and complete unfinished items. To eliminate the potential ceiling effect, Goldstein et al. used a more stringent scoring procedure than raw scores. They counted an item as correct only if both stimuli for the item were correct. With this approach, ratio scores were the proportion of correct items in the total number of items attempted. They scored the short 
Table 1

Effect Sizes $(d)$ and Probability Levels ( $p$ ) From

Previous Studies of the Effects of Performance Factors on the Gender Difference on the MRT

\begin{tabular}{cllll}
\hline \multicolumn{1}{c}{ Investigation } & Condition & \multicolumn{1}{c}{$\begin{array}{c}\text { Scoring } \\
\text { Procedure }\end{array}$} & $d$ & $p$ \\
\hline Goldstein et al. $(1990$, & Short time & raw score & .85 & .001 \\
Experiment $1, N=68)$ & & ratio score & .29 & .25 \\
Goldstein et al. $(1990$, & Short time & stringent score & .83 & .01 \\
Experiment $2, N=46)$ & & stringent ratio score & .56 & .10 \\
& Untimed & stringent score & .60 & .06 \\
Stumpf $(1993, N=233)$ & Short time & raw score & .74 & .001 \\
& & ratio score & .48 & .001 \\
Resnick $(1993, N=182)$ & Unlimited time & raw score & .75 & .0001 \\
& & stringent score & .80 & .0001 \\
& & corrected score & .74 & .0001 \\
\hline
\end{tabular}

Note-Raw score, number of correct stimuli; ratio score, proportion of correct stimuli in the number of stimuli attempted; stringent score, number of correct items; stringent ratio score, proportion of correct items in the number of items attempted; corrected score, number of correct stimuli minus number of incorrect stimuli.

time portion of the test with both stringent scores and the stringent ratio scores. They also used the stringent score procedure to create a total score for an "untimed" condition. This score consisted of the sum of the stringent scores for the items completed during the short time period and the items completed during the additional time interval.

As can be seen in Table 1, Goldstein et al. (1990) found a significant gender difference for stringent scores in the short time condition, the effect size of which was comparable to that found with raw scores in their first study. Rather than finding no indication of a gender difference for stringent ratio scores in the short time condition and for stringent scores in the untimed condition, they found a marginally significant gender difference for each condition. The effect sizes of the gender difference for stringent ratio scores in the short time condition and stringent scores in the untimed condition were larger than those found with ratio scores in their first study (see Table 1). Given these effect sizes, Goldstein et al. may have found a significant gender difference in performance in each condition had they used a larger sample size. To find a significant gender difference $80 \%$ of the time with these effect sizes, a sample size of approximately 47 men and 47 women would have been necessary (Cohen, 1988, Table 2.4.1, p. 55). The small sample size used by Goldstein et al. made it unlikely that they would have found a significant gender difference after the time limit and the scoring procedure were manipulated. Rather than eliminating the significant gender difference on the test by manipulating these variables, they may have "eliminated" it by capitalizing on Type II error.

In addition, Goldstein et al. (1990) used analysis of covariance (ANCOVA) to analyze the data from this study, with the subjects' Scholastic Aptitude Test math subtest score as a covariate. This resulted in an elimination of the marginally significant gender difference for stringent ratio scores in the short time condition and for stringent scores in the untimed condition. The gender difference for strin- gent scores in the short time condition remained significant, however.

From the results of their first study and those of the ANCOVA, Goldstein et al. (1990) concluded that the gender difference on the MRT disappears when the gender difference in the number of items attempted is controlled either by using ratio scores or when men and women are allowed to complete all items on the test.

Goldstein et al. (1990) contended that earlier research yielded a significant gender difference on the MRT because of the use of raw scores. Yet despite this contention, few investigators of the gender difference on the MRT have actually used raw scores (Block, Arnott, Quigley, \& Lynch, 1989; Casey \& Brabeck, 1989). Both before 1990 and subsequently, most investigators have instead used stringent scores or scored only the items attempted and included a correction for guessing. ${ }^{1}$ Furthermore, they varied the amount of time that subjects were allowed on the MRT. Despite differences in scoring procedures and time limits, each of these studies resulted in a highly significant gender difference on the test.

The hypothesis that the gender difference on the MRT arises because women complete fewer items than men do is not supported by McGee's (1979) item analysis of the test. For each item on the MRT, McGee determined the percentage of men and women who answered the item correctly. He found that for every item women were less accurate than men.

The assertion that the gender difference on the MRT is due to a gender difference in the number of items attempted is not supported by a study performed by Stumpf (1993) published in this journal. Stumpf administered the MRT to 146 male and 87 female adolescents at the Center for Talented Youth and found that the effect size of the gender difference when the tests were scored with raw scores was reduced when ratio scores were used (see Table 1). However, Stumpf found that the gender difference with ratio scores was still significant. 
In a recent study published in this journal, Delgado and Prieto (1996) attempted to replicate Goldstein et al.'s (1990) findings. They administered to over 1,400 Spanish high school students a three-dimensional mental rotations test used to select aircraft pilots in Spain. They allowed the students either 5 or $15 \mathrm{~min}$ on the test. Delgado and Prieto then examined whether the gender difference on their mental rotations test varied as a function of the time limit and the scoring procedure. Contrary to their expectations, the effect size of the gender difference with raw scores did not decrease when the time period was increased. Instead, they found that the gender difference became larger, because girls made proportionally fewer correct responses as they attempted more items. Furthermore, although altering the scoring procedure reduced the magnitude of the overall gender difference from $d=$ .89 with raw scores to $d=.59$ with ratio scores, the investigators found that the significant gender difference did not disappear in either the short time or long time groups. Thus Delgado and Prieto found no evidence on their mental rotations test that the gender difference disappears when the time limit or the scoring procedure is manipulated.

Stumpf (1993) on the MRT and Delgado and Prieto (1996) on their mental rotations test found, consistent with Goldstein et al. (1990), that women attempted fewer items than men did when the tests were timed. They also found that when ratio scores rather than raw scores were used to control the gender difference in the number attempted, the magnitude of the gender difference in performance on the MRT was reduced. However, the use of ratio scores to control the gender difference in the number of items attempted is questionable. Ratio scores will tend to favor individuals who attempt fewer items over those who attempt more items. An individual who completes only a few items but gets them correct will receive a higher score than an individual who completes all the items but errs on a few.

Rather than use ratio scores to control for the gender difference in the number of items attempted, one can simply allow men and women unlimited time to complete the entire test. This has been done. Resnick (1993) administered the MRT to 88 male and 94 female college students and allowed them as much time as they needed to complete all of the items on the test. She then scored the test in three ways: the number of correct stimuli, the number of correct items, and the number of correct stimuli minus the number of incorrect stimuli. She found that, regardless of the scoring procedure, men scored significantly higher than women on the test, and the effect size of the gender difference for each scoring method was large (see Table 1).

In summary, the published studies do not support the hypothesis that the gender difference on the MRT is due to a gender difference in the number of items attempted. Furthermore, the disappearance of the significant gender difference on the MRT in Goldstein et al.'s (1990) study can be explained by Type II error. Thus they did not adequately test whether the gender difference actually dis- appears when the gender difference in the number of items attempted is controlled.

Resnick's (1993) study showed that the gender difference on the MRT does not disappear when women and men are allowed sufficient time to complete the entire test. However, she did not address the question of whether the gender difference is larger with a short time limit than with unlimited time-that is, whether women's scores suffer differentially when the time limit is short. The present experiment was designed in order to investigate this question.

To determine whether the gender difference changes as a function of the time limit, men and women were allowed either a short time or unlimited time to perform on the test. To determine whether the gender difference changes as a function of the scoring procedure, all of the tests were scored with the three scoring procedures used by Resnick (1993). In addition, the effect of ratio scoring on the gender difference in performance in the short time condition was examined.

\section{METHOD}

\section{Subjects}

The subjects were 70 male and 70 female college students enrolled in undergraduate psychology courses at San Jose State University. All were between the ages of 17 and 25 years old $(M=$ 20.7). All received experimental credits for participating.

\section{Materials}

The MRT (Vandenberg, 1975) is a pencil-and-paper version of the chronometric task originally devised by Shepard and Metzler (1971). The MRT requires subjects to recognize three-dimensional block stimuli that are the same as a target figure, but rotated in three-dimensional space. The test consists of two parts with 10 items/ part. For each item, a target stimulus is presented, and the subjects are asked to select from four alternatives the two stimuli that are identical to the target stimulus.

\section{Procedure}

The subjects were randomly assigned to either a short time or an unlimited time condition, with the constraint that each condition have equal numbers of men and women. There were 35 men and 35 women in the short time group and 35 men and 35 women in the unlimited time group.

Subjects in the short time condition were allowed $3 \mathrm{~min}$ for each of the two parts of the test. This time limit was used by Goldstein et al. (1990) in their short time condition. In the unlimited time condition, the subjects were allowed as much time as they needed to completely answer all items in each part of the test. These subjects completed all items in each part in 9 min or less.

Three scoring procedures were used for all tests: the number of correct stimuli, or raw score; the number of correct items, or stringent score; and the number of correct stimuli minus the number of incorrect stimuli, or corrected score. Also tests in the short time condition were scored with ratio scores.

\section{RESULTS AND DISCUSSION}

Figure 1 shows the mean scores of women and men for each of the three scoring methods in the two groups. These data indicate that, regardless of the scoring method used, men scored higher than women. This was true in 

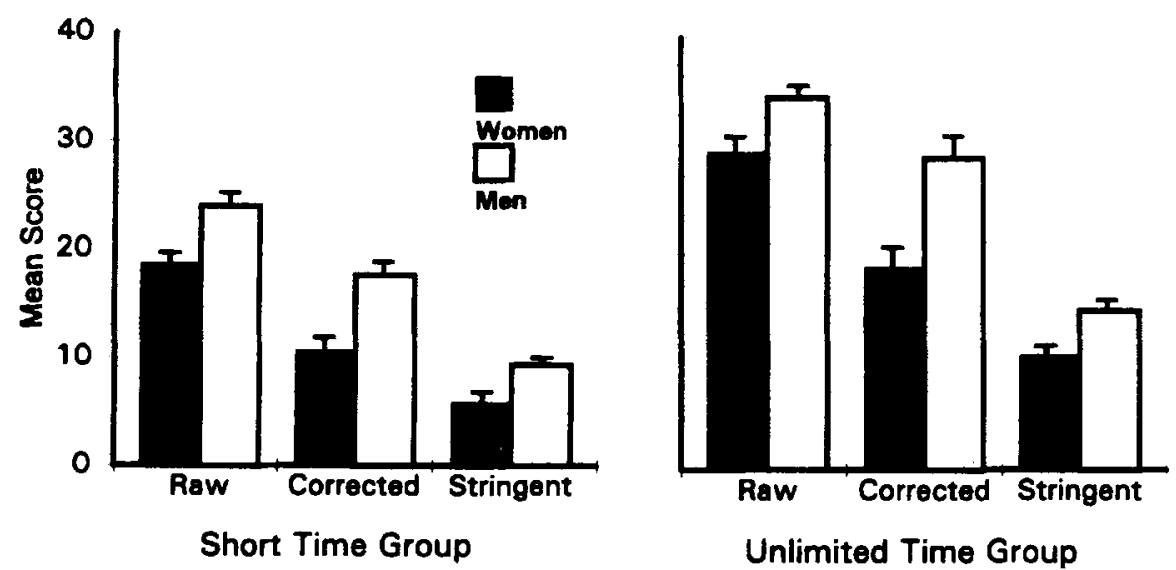

Figure 1. Mean scores and standard errors on the MRT as a function of gender and scoring method in the two time groups. The maximum score using the raw score and the corrected score procedures is $\mathbf{4 0}$ points. The maximum score using the stringent score procedure is $\mathbf{2 0}$ points.

both the short time group and the unlimited time group (in which men and women completed the entire test). Thus there is no evidence to support the hypothesis that the gender difference on the MRT is due to the scoring method or to a gender difference in the number of items (or stimuli) attempted. A two-way (gender $\times$ time limit) analysis of variance performed on each of the three types of scores supports these conclusions.

Cohen's $d$ was calculated for the gender difference in each time condition for each scoring procedure. These $d$ s, shown in Table 2, were comparable to those found in previous studies of the gender difference on the MRT (Masters \& Sanders, 1993).

Table 2 also shows the results of chi-square analyses (Rosenthal \& Rubin, 1982) that tested whether there was any difference between the $d \mathrm{~s}$ in each time condition for each of the three types of scores. There was no significant difference between the $d$ s for any of the scores. Thus there was no evidence that altering the time limit affected the magnitude of the gender difference on the MRT.

Goldstein et al. (1990) and Stumpf (1993) on the MRT and Delgado and Prieto (1996) on their mental rotations test found that women attempted fewer stimuli than men did when the tests were timed. A one-tailed independent $t$ test performed on the number of stimuli attempted in my short time condition indicated that women did indeed attempt fewer stimuli $(M=26.8)$ than men $\operatorname{did}(M=30.4)$ $[t(68)=1.92, p<.05]$. But the gender difference on the MRT cannot be due to the gender difference in the num-

Table 2

Chi-Square Tests of the Effect Sizes $(d)$ in the Short and Unlimited Time Groups for the Three Types of Scores

\begin{tabular}{|c|c|c|c|c|}
\hline & Short Time & $\begin{array}{l}\text { Unlimited } \\
\text { Time } \\
\end{array}$ & & \\
\hline & $d$ & $d$ & $\chi^{2}(1)$ & $p$ \\
\hline Raw score & .86 & .96 & .08 & .80 \\
\hline Stringent score & .99 & .94 & .02 & .80 \\
\hline Corrected score & .84 & .96 & .11 & .70 \\
\hline
\end{tabular}

ber of stimuli attempted. Regardless of the scoring procedure, when men and women completed all items in the unlimited time condition there was still a highly significant gender difference, and the magnitude of this difference was the same as that in the short time condition.

I also examined whether scoring the tests with ratio scores affects the gender difference on the MRT. Because ratio scores tend to favor individuals who attempt fewer stimuli and because women consistently attempt fewer stimuli than men on the MRT, the gender difference on the test should be smaller with ratio scores than with other scoring procedures. To test this hypothesis, I first examined whether attempting fewer stimuli is associated with a higher test score. Because subjects in the unlimited time condition completed all items, ratio scores were calculated only for the tests of the 70 subjects in the short time condition. The tests in this condition were scored with ratio scores (the proportion of correct stimuli in the total number of stimuli attempted), and these scores were correlated with the number of stimuli attempted on the test. The results of this analysis showed that as the number of stimuli attempted decreased, ratio scores increased for both men $[r(33)=-.26]$ and women $[r(33)=--.40] .^{2}$ Thus, attempting fewer stimuli is associated with an increased score.

A two-tailed independent $t$ test performed on the ratio scores showed that men $(M=.80)$ performed significantly better than women $(M=.72)[t(68)=2.47, p<$ $.02]$. But the use of ratio scores resulted in a smaller gender difference $(d=.60)$ than that found with any other scoring procedure in either the short time condition or, more importantly, the unlimited time condition, in which there was no gender difference in the number of stimuli attempted (see Table 2). It appears that the use of ratio scores artificially decreases the gender difference on the test because individuals who attempt fewer stimuli (usually women) tend to receive higher scores than they otherwise would. The smaller effect size of the gender difference on the MRT when ratio scores are used appears 
to be an artifact of that scoring method rather than an actual reduction of the gender difference in performance.

The results of the present experiment failed to support Goldstein et al.'s (1990) hypothesis of a disappearance of the gender difference on the MRT when the time limit is eliminated. There was no evidence that the magnitude of the gender difference even changes as a function of time limit. Instead, I found for each of the three scoring procedures that men scored significantly higher than women in both time conditions and that the magnitude of the gender difference persisted undiminished in the unlimited time condition. Thus, there is no evidence that women's performance suffers differentially when a short time limit is used in investigations of the gender difference on the MRT, nor is there evidence that the scoring procedure is responsible for the gender difference on the test.

The male advantage in performance found in the present experiment is consistent with the results of previous investigations of the gender difference on the MRT. Given that a large gender difference is consistently found on the MRT and that manipulating the time limit and the scoring procedure does not eliminate this difference, I believe that it is time to abandon the hypothesis that performance factors account for the gender difference on the test.

\section{REFERENCES}

BEATTY, W. W., \& DunCan, D. (1990). Relationship between performance on the Everyday Spatial Activities Test and on objective measures of spatial behavior in men and women. Bulletin of the Psychonomic Society, 28, 228-230.

Block, R. A., ARnott, D. P., Quigley, B., \& LYNCH, W. C. (1989). Unilateral nostril breathing influences lateralized cognitive performance. Brain \& Cognition, 9, 181-190.

Bouchard, T. J., \& McGeE, M. G. (1977). Sex differences in human spatial ability: Not an X-linked recessive gene effect. Social Biology, 24, 332-335

CASEY, M. B., \& BrabeCK, M. M. (1989). Exceptions to the male advantage on a spatial task: Family handedness and college major as factors identifying women who excel. Neuropsychologia, 27, 689-696

Casey, M. B., Colon, D., \& GorIs, Y. (1992). Family handedness as a predictor of mental rotation ability among minority girls in a mathscience training program. Brain \& Cognition, 18, 88-96.

COHEN, J. (1988). Statistical power analysis for the behavioral sciences (2nd ed.). Hillsdale, NJ: Erlbaum.

Delgado, A. R., \& Prieto, G. (1996). Sex differences in visuospatial ability: Do performance factors play such an important role? Memory \& Cognition, 24, 504-510.

FreEdMAN, R. J., \& Rovegno, L. (1981). Ocular dominance, cognitive strategy, and sex differences in spatial ability. Perceptual \& Motor Skills, 52, 651-654

Geary, D. C., Gilger, J. W., \& Elliott-Miller, B. (1992). Gender differences in three-dimensional mental rotation: A replication. Journal of Genetic Psychology, 153, 115-117.

Goldstein, D., Haldane, D., \& Mitchell, C. (1990). Sex differences in visual-spatial ability: The role of performance factors. Memory \& Cognition, 18, 546-550.

HALPERN, D. (1992). Sex differences in cognitive abilities (2nd ed.). Hillsdale, NJ: Erlbaum.

LinN, M. C., \& Petersen, A. C. (1985). Emergence and characteriza- tion of sex differences in spatial ability: A meta-analysis. Child Development, 56, 1479-1498.

LiPPA, R., \& CONNELLY, S. (1990). Gender diagnosticity: A new Bayesian approach to gender-related individual differences. Journal of Personality \& Social Psychology, 59, 1051-1065.

MaccoBY, Е. E., \& JACKLIN, C. N. (1974). The psychology of sex differences. Stanford, CA: Stanford University Press.

Masters, M. S., \& Sanders, B. (1993). Is the gender difference in mental rotation disappearing? Behavior Genetics, 23, 337-341.

MCGeE, M. G. (1979). Human spatial abilities: Sources of sex differences. New York: Praeger.

OZER, D. J. (1987). Personality, intelligence, and spatial visualization: Correlates of Mental Rotations test performance. Journal of Personality \& Social Psychology, 53, 129-134.

Pearson, J. L., \& Ferguson, L. R. (1989). Gender differences in patterns of spatial ability, environmental cognition, and math and English achievement in late adolescence. Adolescence, 24, 421-431.

ResNicK, S. M. (1993). Sex differences in mental rotations: An effect of time limits? Brain \& Cognition, 21, 71-79.

RosenthaL, R., \& RuBiN, D. B. (1982). Comparing effect sizes of independent studies. Psychological Bulletin, 92, 500-504.

SANDERS, B., \& SOARES, M. P. (1986). Sexual maturation and spatial ability in college students. Developmental Psychology, 22, 199-203

Sanders, B., Soares, M. P., \& D'Aquila, J. M. (1982). The sex difference on one test of spatial visualization: A nontrivial difference. Child Development, 53, 1106-1110.

ShePARD, R., \& METZLER, J. (1971). Mental rotation of three-dimensional objects. Science, 171, 701-703.

STUMPF, H. (1993). Performance factors and gender-related differences in spatial ability: Another assessment. Memory \& Cognition, 21, 828-836

VANDENBERG, S. G. (1975). Sources of variance in performance on spatial tests. In J. Eliot \& N. J. Salkind (Eds.), Children's spatial development (pp. 57-66). Springfield, IL: Charles C. Thomas.

VANDENBERG, S. G., \& Kuse, A. R. (1978). Mental Rotations, a group test of three-dimensional spatial visualization. Perceptual \& Motor Skills, 47, 599-604.

VANDENBERG, S. G., KuSE, A. R., \& Vogler, G. P. (1985). Searching for correlates of spatial ability. Perceptual \& Motor Skills, 60, 343-350.

WILSON, J. R., \& VANDENBERG, S. G. (1978). Sex differences in cognition: Evidence from the Hawaii family study. In T. E. McGill, D. A. Dewsbury, \& B. D. Sachs (Eds.), Sex and behavior: Status and prospectus (pp. 317-335). New York: Plenum.

YEN, W. M. (1975). Sex-linked major-gene influences on selected types of spatial performance. Behavior Genetics, 5, 281-298.

\section{NOTES}

1. See Beatty and Duncan, 1990; Bouchard and McGee, 1977; Casey, Colon, and Goris, 1992; Freedman and Rovegno, 1981; Geary, Gilger, and Elliott-Miller, 1992; Lippa and Connelly, 1990; Ozer, 1987; Pearson and Ferguson, 1989; Sanders and Soares, 1986; Sanders et al., 1982; Vandenberg and Kuse, 1978; Vandenberg, Kuse, and Vogler, 1985; Wilson and Vandenberg, 1978; Yen, 1975.

2. I also calculated stringent ratio scores (the proportion of correct items in the total number of items attempted) for the tests in the short time group. The results for these scores were the same as those with ratio scores: men scored significantly higher than women, the effect size of the gender difference was smaller than that of other scoring methods in either time condition, and the correlation between stringent ratio scores and the number of items attempted was negative for both men and women.

(Manuscript received November 11, 1996; revision accepted for publication March 10, 1997.) 